\title{
ORDER SUMMABILITY AND ALMOST CONVERGENCE
}

\author{
HOWARD T. BELL
}

\begin{abstract}
The object of this paper is to compare the summability methods of almost convergence and order summability. This is done by examining a method of summability which includes both methods. The main result is that every monotone Fourier effective matrix is strongly regular.
\end{abstract}

Introduction. In this paper a general, nonmatrix method of summability is introduced which not only includes matrix summability but also includes the order summability of Jurkat and Peyerimhoff [2] and the method of almost convergence of G. G. Lorentz [5]. Some general theorems about this method are given and they are used to examine the relationship between the two methods mentioned above and the relationship between almost convergence and Fourier effectiveness. We are able to show that every monotone Fourier effective matrix is strongly regular.

In $\S 1$, we define the method and give some examples. The study of the mapping properties of this method comprise $\S 2$. As applications of this method, comparison theorems between order summability and almost convergence are proved in $\S 3$.

1. Definitions and examples. For $v=1,2, \cdots$, let $A^{v}=\left(a_{n k}^{v}\right)$ be an infinite matrix of complex (or real) numbers. Let $\mathscr{A}$ denote the sequence of matrices $\left\{A^{v}\right\}$. For a sequence of complex (or real) numbers, $x=\left\{x_{n}\right\}$, the double sequence $t=\left\{t_{n}^{v}\right\}$, defined by $t_{n}^{v}=\sum_{k=1}^{\infty} a_{n k}^{v} x_{k}$ is called the $\left\{A^{v}\right\}$ or $\mathscr{A}$-transform of $x$ whenever the series converges for all $n$ and $v$. A sequence $x$ is said to be $\left\{A^{v}\right\}$ or $\mathscr{A}$-summable to some number $L$ if $\left\{t_{n}^{v}\right\}$ converges to $L$ as $n$ tends to $\infty$ uniformly for $v=1,2, \cdots . L$ is said to be the $\mathscr{A}$-limit of $x$, written $\lim _{\mathscr{A}} x=L$ or $\mathscr{A} x \rightarrow L$, and we say $\mathscr{A} x$ is convergent to $L$.

Also, let $c_{\mathscr{d}}$ be the set of all sequences whose $\mathscr{A}$-transform is convergent. $\mathscr{A}$ is said to be a regular sequence of matrices if $x \rightarrow L$ implies $\mathscr{A} x \rightarrow L$. We shall denote the set of null sequences by $n$ and the bounded sequences by $m$.

Received by the editors April 25, 1972.

AMS (MOS) subject classifications (1970). Primary 40C99, 40D05, 40G05; Secondary 40D25, 42A20.

Key words and phrases. Almost convergence, order summability, Fourier effective, $\mathscr{A}$-summability, Silverman-Toeplitz theorem, strongly regular, almost strongly regular.

(c) American Mathematical Society 1973 
We shall now consider some examples. Clearly if, for some matrix $B$, $A^{v}=B$ for $v=1,2, \cdots$, then $\left\{A^{v}\right\}$-summability is just matrix summability by $B$.

Next, consider the sequence of matrices, $\mathscr{F}=\left\{F^{v}\right\}$, with $F^{v}=\left(a_{n k}^{v}\right)$ where $a_{n k}^{v}=1 / n$ if $v \leqq k<n+v$ and is 0 otherwise. Then a sequence $x$ is $\mathscr{F}$ summable to $L$ if $\left(F_{x}^{v}\right)_{n}=(1 / n) \sum_{k=v}^{n+v-1} x_{k}$ converges to $L$ as $n \rightarrow \infty$ uniformly for $v=1,2, \cdots$, that is, if $x$ is almost convergent (see [5]) to $L$. Thus $c_{\mathscr{F}}=f$, the space of almost convergent sequences. Lorentz in [5] has shown that $f$ is not the bounded convergence domain of any regular matrix, but we see that it is the convergence domain of a regular sequence of matrices. In $\S 2$, we will try to show how this facilitates the study of almost convergence.

In [5], Lorentz considers $F_{A^{-}}$-summability and in [6], Mazhar and Siddiqi consider $A_{B}$-summability. Both methods are easily seen to be special cases of $\mathscr{A}$-summability for appropriately chosen $\mathscr{A}$.

In [2], Jurkat and Peyerimhoff define order summability [ $g$ ]. For $g(t)$, defined on $[0,1)$ with $g(t) \geqq 0$, a sequence $x=\left\{x_{n}\right\}$ is said to be order summable $[g]$ to $L$ if

$$
\frac{1}{n+1-m} \sum_{v=m}^{n} x_{v}-L=o\left(1+g\left(\frac{m}{n+1}\right)\right)
$$

as $n \rightarrow \infty$ uniformly for $0 \leqq m \leqq n$.

Let $\mathscr{G}=\left\{G^{v}\right\}$ with $G^{m}=\left(a_{n v}^{m}\right)$ where for each $n, m \leqq n$,

$$
a_{n v}^{m}=1 /(1+g(m /(n+1)))(n+1-m)
$$

for $m \leqq v \leqq n$ and is zero elsewhere in the $n$th row (note that this completely defines $G^{0}$ ) and when $n<m$, the $n$th row of $G^{m}$ is the $n$th row of $G^{0}$. Then $\mathscr{G}$-summability is equivalent to order summability $[g]$.

When dealing with order summability, indices will start at 0 instead of 1 . Also, the sums throughout this paper will be taken from $k=1$ to $\infty$ unless otherwise noted.

2. General theorems. The following notion enables us to reduce some questions on $\mathscr{A}$-summability to similar questions on matrix summability.

THEOREM 1. $c_{S^{\prime}}=\bigcap\left\{c_{U}: U \in \mathscr{U}\right\}$ where $\mathscr{U}$ is the family of all matrices whose nth row is the nth row of $A^{v}$ for some $v$.

Proof. Let $U \in \mathscr{U}$. The $n$th row of $U$ is the $n$th row of $A^{v}$ for some $v$. For that $n$, we shall denote such a $v$ by $v_{n}$. Thus, for each $U \in \mathscr{U}$ we associate a sequence of positive integers $\left\{v_{n}\right\}$ where $u_{n k}=a_{n k}^{v_{n}}$ for all $n$ and $k$.

Now, suppose $x \in c_{s /}$. Then surely $\sum a_{n k}^{v_{n}} x_{k}$ converges as $n \rightarrow \infty$ since $\sum a_{n k}^{v} x_{k}$ converges uniformly for $v=1,2, \cdots$ as $n \rightarrow \infty$. On the other hand, 
suppose $x \notin c_{\mathscr{c}}$. Then, for any $L$ and $\varepsilon>0$, there is an infinite set of integers, $J$, such that for each $n \in J$, there is an integer $w_{n}$ such that $\left|\sum a_{n k}^{w_{n}} x_{k}-L\right|>\varepsilon$ for all $n \in J$. Let $v_{n}=w_{n}$ for $n \in J$ and let $v_{n}=1$ for $n \notin J$. If $U$ is the matrix associated with the sequence $\left\{v_{n}\right\}$, then $U \in \mathscr{U}$ but $U x$ does not converge. Hence, if $x \notin c_{\mathscr{S}}$, then there is a $U \in \mathscr{U}$ with $x \notin c_{U}$.

The consistency of all the $U \in \mathscr{U}$ and $\mathscr{A}$ also follows from this assertion. For if the $U$-limit of $x$ differed from the $V$-limit of $x$ for some $U, V \in \mathscr{U}$, we could construct a matrix $W \in \mathscr{U}$ by alternating the rows of $U$ and $V$, where the $W$-limit of $x$ would not exist. Hence, as corollaries, we have that $\mathscr{A}$ is regular (resp., conservative, coercive, strongly regular, etc.) if and only if each $U \in \mathscr{U}$ is regular (resp., conservative, coercive, strongly regular, etc.).

The proofs of the following three theorems are omitted. (Complete proofs can be found in [1].) The proof of each of the theorems follows the same pattern. Suppose we wish to show that $\mathscr{A}$ is, say, regular if and only if (a), (b) and (c) hold. The classical "Silverman-Toeplitz" theorem tells us that $U$ is regular if and only if (i), (ii) and (iii) hold for $U$. Thus the proof reduces to showing (a), (b) and (c) hold if and only if (i), (ii) and (iii) hold for every $U \in \mathscr{U}$.

THEOREM 2. A sequence of matrices $\mathscr{A}=\left\{A^{v}\right\}$ is regular if and only if

(a) for each $k=1,2, \cdots, \lim _{n \rightarrow \infty} a_{n k}^{v}=0$ uniformly for $v=1,2, \cdots$;

(b) $\lim _{n \rightarrow \infty} \sum a_{n k}^{v}=1$ uniformly for $v=1,2, \cdots$;

(c) for each $n, v=1,2, \cdots, \sum\left|a_{n k}^{v}\right|<\infty$, and there exists integers $N$, $M$ such that $\sum\left|a_{n k}^{v}\right|<M$ for $n \geqq N$ and all $v=1,2, \cdots$.

A sequence of matrices $\mathscr{A}$ (or a matrix $A$ ) is called strongly regular if, whenever a sequence $x$ is almost convergent to $L$, then $\mathscr{A} x$ (or $A x$ ) converges to $L$. We have the following analog to the characterization of strongly regular matrices given by Lorentz [5].

THEOREM 3. A regular sequence of matrices $\mathscr{A}=\left\{A^{v}\right\}$ is strongly regular if and only if $\lim _{n \rightarrow \infty} \sum\left|a_{n, k+1}^{v}-a_{n, k}^{v}\right|=0$ uniformly for $v=1,2, \cdots$.

THEOREM 4. A sequence of matrices $\mathscr{B}=\left\{B^{v}\right\}$ maps $m$ to $n$, that is, the $\mathscr{B}$-transform of every bounded sequence converges to 0 if and only if $\sum\left|b_{n k}^{v}\right| \rightarrow 0$ as $n \rightarrow \infty$ uniformly for $v=1,2, \cdots$.

By the following two examples, we try to establish the advantage of considering almost convergence as a special case of a more general situation. A matrix $A$ is almost regular if, whenever $x \rightarrow L$ then $A x$ is almost convergent to $L$. Now $A x$ is almost convergent to $L$ if and only if the $\left\{F^{v} A\right\}$-transform of $x$ converges to $L$. Thus, $A$ is almost regular if and only if $\left\{F^{v} A\right\}$ is regular. Thus, the characterization of almost regular 
by King [4] follows immediately from Theorem 2 . The same approach can be applied to almost strongly regular matrices. If whenever $x$ is almost convergent to $L$, then $B x$ is called almost convergent to $L$, then $B$ is called almost strongly regular. Thus $B$ is such a matrix only if $\left\{F^{v} B\right\}$ is strongly regular. Applying Theorem 3, we obtain the characterization given by Schaefer [7].

We are now able to deduce a useful theorem to determine when a sequence of matrices is strongly regular. Let $S$ denote the matrix with 1 on the super diagonal, i.e., $S=\left(\rho_{n k}\right)$ where $\rho_{n n+1}=1$ and is 0 elsewhere. Also, $I$ will denote the identity matrix.

THEOREM 5. A regular sequence $\mathscr{A}$ is strongly regular if and only if $\mathscr{B}=\left\{B^{v}\right\}$, where $B^{v}=A^{v}(S-I)$, maps $m$ to $n$.

Proof. We first note that for any $v$, the $n, k$ th entry in $A^{v} S$, which we shall denote by $\left(A^{v} S\right)_{n k}$ is $\left(A^{v}\right)_{n, k+1}$ or $a_{n, k+1}^{v}$. Hence, we see that

$$
\sum\left|b_{n k}^{v}\right|=\sum\left|\left(A^{v} S-A^{v}\right)_{n k}\right|=\sum\left|a_{n, k+1}^{v}-a_{n k}^{v}\right| .
$$

Applying Theorems 3 and 4 establishes the result.

3. Comparison theorems. In certain cases we are able to compare order summability and almost convergence.

THEOREM 6. Suppose $g(t)$ is defined on $[0,1)$ and $g(t) \geqq 0$. If $\lim _{t \rightarrow 1} g(t)=$ $\infty$, then order summability $[g]$ is stronger than almost convergence, i.e., is strongly regular.

Proof. We first note that order summability $[g]$ is regular and since order summability $[g]$ is $\mathscr{G}$-summability (see $\S 1$ ), we can apply Theorem 5 . Thus, we must show that if $x \in m$, then $((S-I) x)_{v}=\left\{x_{v+1}-x_{\imath}\right\}$ is $\mathscr{G}$ summable or order summable $[g]$ to 0 . We first note that

$$
\left|\sum_{r=m}^{n}\left(x_{v+1}-x_{v}\right)\right|=\left|x_{n+1}-x_{m}\right| \leqq 2\|x\| .
$$

Let $\varepsilon>0$ be given. Since $\lim _{t \rightarrow 1} g(t)=\infty$, we can choose $\delta, 0<\delta<\frac{1}{2}$ such that $1-m /(m+1)<\delta$ implies $g(m /(n+1))>2\|x\| / \varepsilon$. Also, choose $N$ so that $n \geqq N$ implies $1 /(n+1) \delta<\varepsilon / 2\|x\|$.

Then for $n \geqq N$ and $0 \leqq m \leqq n$, if $1-\delta<n /(n+1)$ we have

$$
1 /(1+g(m /(n+1)))<\varepsilon / 2\|x\|
$$

or if $1-\delta>m /(n+1)$, then

$$
1 /(n+1-m)<1 /(n+1) \delta<\varepsilon / 2\|x\|,
$$


thus

$$
\frac{1}{1+g\left(\frac{m}{n+1}\right)} \cdot \frac{1}{n+1-m}\left|\sum_{v=m}^{n} x_{v+1}-x_{v}\right|<\varepsilon / 2\|x\| \cdot 2\|x\|=\varepsilon
$$

for $n>N$ and $0 \leqq m \leqq n$.

COROllaRy 1. Order summability $[\log (1 /(1-t)]$ is strongly regular.

COROLlARY 2. Every monotone Fourier effective matrix is strongly regular.

Proof. Jurkat and Peyerimhoff have shown in [2] that every monotone Fourier effective method is stronger than order summability $[\log 1 /(1-t)]$ which is strongly regular by Corollary 1.

Similar theorems can be derived concerning more general functions $g$, and almost convergence using the comparison theorems of Jurkat and Peyerimhoff in [3].

\section{REFERENCES}

1. H. T. Bell, $\mathscr{S}$-summability, Dissertation, Lehigh University, Bethlehem, Pa., 1971.

2. W. B. Jurkat and A. Peyerimhoff, Fourier effectiveness and order summability, J. Approximation Theory 4 (1971), 231-244. MR 43 \#7855.

3. - Inclusion theorems and order summability, J. Approximation Theory 4 (1971), 245-262. MR 43 \#7856.

4. J. P. King, Almost summable sequences, Proc. Amer. Math. Soc. 17 (1966), 12191225. MR 34 \#1752.

5. G. G. Lorentz, A contribution to the theory of divergent sequences, Acta. Math. 80 (1948), 167-190. MR 10, 367.

6. S. M. Mazhar and A. H. Siddiqi, $O n F_{A}$ and $A_{B}$ summability of a trigonometric sequence, Indian J. Math. 9 (1967), 461-466. MR 41 \#4120.

7. P. Schaefer, Matrix transformations of almost convergent sequences, Math. $Z$. 112 (1969), 321-325. MR 40 \#7674.

Department of Mathematics, Lehigh University, Bethlehem, Pennsylvania 18015

Department of Mathematics, Shippensburg State College, Shippensburg, PenNSYlVania 17257 